\title{
Optimierung chirurgischer Behandlungen für trans Personen
}

\author{
Barbara Mijuskovic a,c, Dirk Johannes Schaefer ${ }^{b, c}$, David Garcia Nuñez ${ }^{a, c}$ \\ ${ }^{a}$ Dr. med.; ${ }^{b}$ Prof. Dr. med.; ${ }^{c}$ Departement Plastische, Rekonstruktive, Ästhetische und Handchirurgie, Universitätsspital Basel
}

Zur Verbesserung der Behandlung von trans Personen haben sich in den letzten Jahren internationale Gesellschaften wie die EPATH und WPATH (European bzw. World Professional Association for Transgender Health) formiert. Insbesondere die WPATH erstellt regelmässig Guidelines, welche den behandelnden Ärzt_innen die aktuellsten Behandlungsanweisungen vermitteln [3]. Allerdings existiert zurzeit wenig Konsens bezüglich der möglichen chirurgischen Behandlungen. Dies führt dazu, dass jedes Behandlungszentrum unterschiedliche Therapieansätze mit abweichendem perioperativem Management anbietet, was zu Verunsicherungen sowohl bei den Behandlungssuchenden als auch bei den Behandelnden führen kann. 31.8.-1.9.2018, Universitäts-
spital Basel.

Um die Vielfalt unterschiedlicher Geschlechter darstellen zu können, werden in diesem Text Pluralformen mit einem «gender gap» (z.B. Patient_ innen) gekennzeichnet. Der gender gap ist als Alternative zum Binnen-I (z.B. PatientInnen) zu verstehen und dient der Öffnung des Raumes für Geschlechter jenseits des binären Konzepts.

\section{Konsensfindung im Bereich der chirurgischen Behandlungen}

Die Anzahl geschlechtsangleichender Operationen hat in den letzten Jahren international und auch in der Schweiz zugenommen [1]. Eine Ursache für diesen Trend besteht in der weltweiten Prävalenzzunahme von trans Personen [2]. Auch aufgrund der zunehmenden Akzeptanz in der Gesellschaft fassen diese vermehrt den Mut zu einer sozialen und medizinischen Transition, welche in vielen Fällen auch operative Massnahmen beinhaltet (Tab. 1). In der Schweiz besteht für die meisten medizinischen Transitionsmassnahmen eine Leistungspflicht der Krankenversicherer.

Tabelle 1: Mögliche geschlechtsangleichende Eingriffe.

\begin{tabular}{ll}
\hline Maskulinisierende Eingriffe & Feminisierende Eingriffe \\
\hline Brustbereich & $\bullet$ Brustkonstruktion \\
\hline - Mastektomie & \\
\hline $\begin{array}{l}\text { Genitalbereich } \\
\text { Kolpsterektomie, Ovarektomie, }\end{array}$ & $\bullet$ Orchiektomie \\
$\begin{array}{l}\text { - Phalloplastik mit/ohne Bildung } \\
\text { von Harnröhre }\end{array}$ & $\bullet$ Penektomie mit Bildung einer Vagina, \\
- Scrotumplastik & Klitoris, Neumündung der Urethra, Vulva \\
\hline Kopf-/Halsbereich & \\
\hline & $\bullet$ Kehlkopfreduktion \\
& $\bullet$ Gesichtsfeminisierende Eingriffe \\
\hline
\end{tabular}

Das Behandlungsteam am Schwerpunkt für Geschlechtervarianz (SPGV) des Universitätsspitals Basel erachtet es als dringend indiziert, einen verbesserten nationalen Konsens zu etablieren, um den trans Personen optimierte chirurgische Behandlungen - samt Verbesserungen im interdisziplinären perioperativen Management - anzubieten. Zu diesem Zweck organisierten die Autorinnen und Autoren im Namen des Schwerpunkts eine Konsensuskonferenz*.

\section{Wichtige Diskussionspunkte}

Nachfolgend werden die wichtigsten Punkte des Meetings zusammengefasst. In der Online-Ausgabe der schweizerischen Ärztezeitung finden Sie eine ausführliche Version unserer Arbeit mit detaillierteren Angaben zu den Resultaten.

\section{Präoperative Betreuung}

\section{Psychiatrische Diagnostik und Behandlung}

2022 wird der psychiatrische ICD-11 in Kraft treten, worin die Genderdysphorie (GD)/Transsexualismus nicht mehr als Krankheit aufgeführt sein wird. Es besteht noch keine Klarheit darüber, welche Konsequenzen die Entpsychiatrisierung der Diagnostik hervorrufen wird. Die Mehrheit der Konferenzteilnehmenden ist der Meinung, dass eine zweijährige psychiatrische (Zwangs-)Behandlung keine Voraussetzung für die 


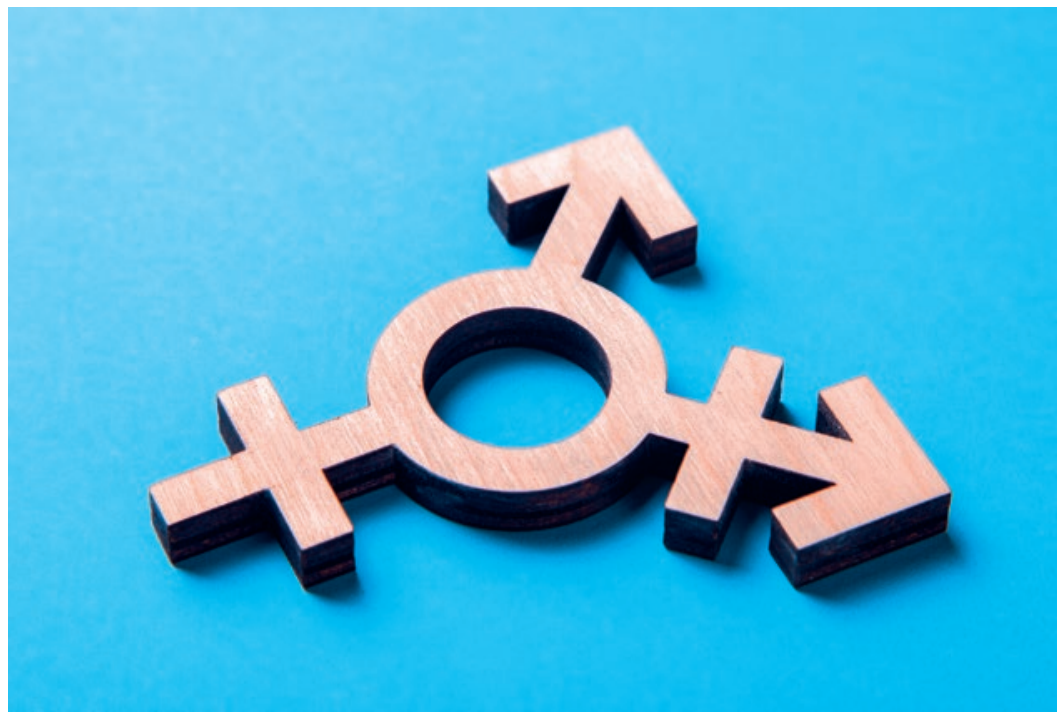

Die Anzahl geschlechtsangleichender Operationen hat in den letzten Jahren international und auch in der Schweiz zugenommen.

Durchführung von geschlechtsangleichenden Operationen darstellen kann. Andererseits sind sich alle Teilnehmenden einig, dass vor der chirurgischen Indikationsstellung eine psychiatrische Beurteilung trotzdem notwendig bleiben wird, nicht zuletzt, weil die trans Bevölkerung ausserordentlichen psychosozialen Risiken ausgesetzt ist, welche zu psychischen Problemen und gelegentlich auch zu perioperativen Konflikten führen können.

\section{Mindestalter für eine chirurgische Geschlechtsangleichung}

Im europäischen Raum wird in der Regel ein Mindestalter von 18 Jahren für eine chirurgische Geschlechtsangleichung empfohlen. Rechtlich ist dies, zumindest in der Schweiz, nirgends festgehalten. Die trans Personen, welche sich klinisch vorstellen, werden zunehmend jünger. Alle Panelist_innen sind sich einig, dass die aktuelle Mindestalter-Empfehlung bei der Mastektomie bei trans Männern nicht zwingend erfüllt sein muss.

\section{Präoperative Hormonbehandlung}

Die Mehrheit der Teilnehmenden an der Konferenz befürwortet eine einjährige Hormontherapie vor der Durchführung geschlechtsangleichender Operationen. In Anbetracht dessen, dass die endokrinologische Behandlung von trans Männern in der Regel nicht zu einer relevanten Veränderung des Brustvolumens führt, sieht die Mehrheit der Panelist_innen die Einhaltung dieses Intervalls bei dieser Subgruppe jedoch als nicht zwingend indiziert. Bis vor wenigen
Jahren wurde das Pausieren der Östrogentherapie vor der genitalen Geschlechtsangleichung bei trans Frauen wegen des Thromboserisikos verlangt. Da viele Patientinnen diese Östrogenpause als negativ bzw. teilweise mit einer depressiven Symptomatik erlebten und im Rahmen anderer antithrombotischer Massnahmen das Risiko als zumutbar betrachtet wird, beurteilt die Mehrheit der Teilnehmenden die Hormonsistierung vor diesem operativen Eingriff als nicht mehr nötig.

\section{Operativ feminisierende Geschlechts- angleichung}

\section{Chirurgische Brustkonstruktion}

Die Konferenzteilnehmenden kommen zu einem Konsens darüber, dass sowohl die Augmentation mit Silikonimplantaten als auch eine Augmentation durch autologe Fettgewebstransplantation wirksam und zweckmässig ist.

\section{Chirurgische Genitalangleichung}

International werden zwei grundlegende Techniken für die genitalangleichende Operation verwendet: die Penisinversionstechnik und die Vaginoplastik mit Darm (Sigmavaginoplastik) [4]. Mit einer Ausnahme bestimmen die Panelist_innen die Penisinversion als die Standardprozedur zur Genitalangleichung. Die Mehrheit der Teilnehmenden der Konferenz sehen die Sigmavaginoplastik für Revisionsoperationen reserviert. Alternativ kann diese Technik in Fällen, wo die primären Geschlechtsorgane keine genügende Länge für eine Penisinversionstechnik vorweisen, angeboten werden.

\section{Chirurgische Nachsorge}

Über die Dauer und Ausgestaltung der postoperativen Nachsorge gibt es aktuell in der Literatur kaum Daten. Mehr als die Hälfte der Panelist_innen findet, dass sich die chirurgische postoperative Nachkontrollperiode über ein Jahr erstrecken sollte. Längere oder gar eine lebenslange chirurgische Nachsorge wird hingegen als unnötig betrachtet. Eine Minderheit der Konferenzteilnehmenden findet, dass sich trans Frauen - wie cis Frauen - postoperativen Mammographien unterziehen sollten. Als unnötig wird erachtet, dass sich trans Frauen identischen PSA-Screeningprozeduren wie cis Männer unterziehen. Mehr als die Hälfte ist aber der Meinung, dass trans Frauen regelmässig gynäkologische Kontrollen durchführen und bei Auffälligkeiten die behandelnde Chirurgin oder den behandelnden Chirurgen konsultieren sollten. 


\section{Interventionen im Gesichtsbereich}

Viele trans Frauen leiden unter geschlechtsdysphorischen Symptomen, welche insbesondere das Gesicht betreffen, und wünschen deshalb geschlechtsangleichende Interventionen in diesem Bereich. Die gesichtsfeminisierende Chirurgie wird zurzeit nur in Ausnahmefällen durch die Krankenkassen übernommen. Das Panel ist mit einer Ausnahme der Meinung, dass weiterhin individuelle Beurteilungen nötig sind, wobei deutlich ausgeprägte, stereotypisch männliche Gesichtszüge, die zu einer Geschlechtsdysphorie führen könnten, korrigiert und durch die Kassen übernommen werden sollten.

\section{Operativ maskulinisierende Geschlechtsangleichung}

\section{Brustentfernung}

Die operative Technik der Mastektomie ist abhängig von der Brustgrösse der zu operierenden trans Person. Bei einer grossen Brust besteht der Konsens bei den Teilnehmenden des Panels, eine Mastektomietechnik mit freier Mamillen-Areolen-Komplex (MAK)-Transplantation anzuwenden. Die Reduktion mit vertikaler oder gar T-förmiger Narbe wird hingegen nicht empfohlen. Die zirkumareoläre Mastektomie mit Narbenpositionierung lediglich um die Brustwarze ist die Standardmethode bei kleiner Brust.

\section{Phalloplastik}

Bei der Durchführung der Phalloplastik werden international unterschiedliche Techniken angewendet. Die in Europa am häufigsten angewendete ist die freie sensibilisierte Radialislappenplastik. Da diese Technik mit gewissen Nachteilen behaftet ist, unter Umständen keine genügende Phallusgrösse gewährleisten kann und insbesondere eine stigmatisierende Narbe am Unterarm hinterlässt [5], sind im Verlauf der Zeit weitere Phalloplastik-Techniken entwickelt worden. Die am zweithäufigsten durchgeführte Technik ist die gestielte antero-lateral thigh (ALT)-Lappenplastik vom Oberschenkel [6]. Diese Technik behebt zwar einige Probleme der Radialislappenplastik, bringt aber mehr Operationsschritte mit sich. Die Mehrheit der Panelist innen sieht die Radialislappen-Technik als Standardeingriff und die ALT-Lappenplastik als valable Alternative. Da Fistelbildungen oder Engstellen in der Neoharnröhre häufige Komplikationen darstellen, besteht der Konsens, dass eine Penispumpe immer der letzte Schritt der genitalen Anpassung beim trans Mann darstellt und dass diese Implantation nicht als «all-in-one»-Operation mit der Phalloplastik kombiniert werden sollte. Eine chirurgische Alternative zur
Phalloplastik ist die Metadoioplastik, auch genannt Klitpen, bei der durch eine lokale Urethraverlängerung eine Art Minipenis konstruiert wird [7]. Aus Sicht der Autoren ist die Durchführung der Phalloplastik in der Schweiz ein zumutbarer Eingriff mit identischen Komplikationsraten im internationalen Vergleich [8].

\section{Vernetzung ist zentral}

Die erste schweizerische Konsensuskonferenz war ein wichtiger Schritt zur nationalen und internationalen Vernetzung von Spezialist innen auf dem Bereich der geschlechtsangleichenden Chirurgie. Im Rahmen dieses Treffens konnten wichtige Aspekte der (peri-)operativen Behandlung von Personen mit einer Genderinkongruenz/Genderdysphorie thematisiert werden. Allerdings konnten aus zeitlichen Gründen manche Aspekte (z.B. die Aufrechterhaltung der Reproduktionsmöglichkeit von trans Personen, postoperative Nachsorge etc.) kaum besprochen werden. Im Rahmen weiterer Konsensusmeetings sollten diese und weitere Aspekte thematisiert werden. Der SPGV ist davon überzeugt, dass die hochkomplexe Betreuung und Behandlung von trans Personen am besten unter einer interdisziplinären Perspektive gelingt [9].

\section{Bildnachweis}

Symbolbild, @ Dragan Andrii | Dreamstime.com

Literatur

1 Weissler JM, Chang BL, Carney MJ, Rengifo D, Messa CA, Sarwer DB u. a. Gender-Affirming Surgery in Persons with Gender Dysphoria: Plast Reconstr Surg. März 2018;141(3):388e-96e.

2 Cheung AS, Ooi O, Leemaqz S, Cundill P, Silberstein N, Bretherton I, u.a. Sociodemographic and Clinical Characteristics of Transgender Adults in Australia. Transgender Health. Dezember 2018;3(1):229-38.

3 Schechter LS, D'Arpa S, Cohen MN, Kocjancic E, Claes KEY, Monstrey S. Gender Confirmation Surgery: Guiding Principles. J Sex Med. Juni 2017;14(6):852-6.

4 Selvaggi G, Ceulemans P, De Cuypere G, VanLanduyt K, Blondeel P, Hamdi M, u. a. Gender Identity Disorder: General Overview and Surgical Treatment for Vaginoplasty in Male-to-Female Transsexuals: Plast Reconstr Surg. November 2005;116(6):135e-45e.

5 Van Caenegem E, Verhaeghe E, Taes Y, Wierckx K, Toye K, Goemaere S, u. a. Long-Term Evaluation of Donor-Site Morbidity after Radial Forearm Flap Phalloplasty for Transsexual Men. J Sex Med. Juni 2013;10(6):1644-51.

6 Xu KY, Watt AJ. The Pedicled Anterolateral Thigh Phalloplasty. Clin Plast Surg. Juli 2018;45(3):399-406.

7 Djordjevic ML, Stanojevic D, Bizic M, Kojovic V, Majstorovic M, Vujovic $\mathrm{S}$, u. a. ORIGINAL RESEARCH-INTERSEX AND GENDER IDENTITY DISORDERS: Metoidioplasty as a Single Stage Sex Reassignment Surgery in Female Transsexuals: Belgrade Experience. J Sex Med. Mai 2009;6(5):1306-13.

8 Bauquis O, Decrouy V, Guerid S. Geschlechtsangleichende Chirurgie. Swiss Med Forum - Schweiz Med-Forum [Internet] 2. Dezember 2014 [zitiert 2. Juli 2019];14(49). Verfügbar unter: doi.emh.ch/smf.2014.02100

9 Die Transition des Gesundheitssystems. Vom Cis-Heterozentrismus zur Affirmation der Geschlechtervarianz. Garcia Nuñez, D. Meier, Ch., Schaefer, D. J. 2019. In: Trans \& Care-Trans Personen zwischen Selbstsorge, Fürsorge und Versorgung. M. Appenroth und M. d. M. Castro Varela (Hg.). Transcript Verlag. 